\title{
INFLUENCIA DEL EJERCICIO EN NIÑOS ASMATICOS CON OBESIDAD Y SOBREPESO EN EL HOSPITAL HONORIO DELGADO-AREQUIPA
}

\section{INFLUENCE OF EXERCISE IN CHILDREN WITH ASTHMA WITH OBESITY AND OVERWEIGHT AT HOSPITAL HONORIO DELGADO-AREQUIPA}

\author{
Ronald Molina Suclly ${ }^{1}$ \\ https://orcid.org/0000-0002- 9981-0657

\begin{abstract}
RESUMEN
Introducción: El Asma bronquial puede ser clasificada, en inducida por el ejercicio físico (AIE) y no inducida por el ejercicio físico (ocasionado por otras causas). Objetivo: Determinar la influencia del ejercicio respiratorio en los niños con Asma Persi stente con Obesidad y Sobrepeso. Métodos: Se diseñó un estudio de casos y controles, prospectivo y longitudinal; fue realizado en Consultorio de Externo de Neumología Pediátrica del Servicio de Pediatría del Hospital Regional Honorio Delgado; donde fueron diagnosticados 166 niños de 6 a 11 años con Asma Bronquial persistente, los que fueron repartidos en 2 grupos por igual; cumpliendo el protocolo 25 niños en el grupo de realizo ejercicios respiratorios y 52 niños en el grupo que solo recibieron tratamiento con Corticoides inhalados. Resultados: En los niños participantes predominó el sexo masculino, con una edad media de 7.3 años, predominando la clase de As ma persistente moderada y la mayoría tenía un estado nutricional normal; en la Prueba de broncoprovocación inducida por ejercicio hubo una mejoría similar entre el grupo de que realizaron un programa de ejercicios respi ratorios y los que no los realiza ron. Conclusión: El ejercicio respiratorio mejoraría la función pulmonar en niños asmáticos con sobrepeso $(\mathrm{t}=0.2835, \mathrm{p}=0.777)$
\end{abstract}

Palabras clave: sobrepeso, obesidad, asma bronquial, niños.

\section{ABSTRACT}

Introduction: Bronchial asthma can be classified, as induced by physical effort (EIA) and not induced by physical effort (caused by other causes). Objective: to determine the

\footnotetext{
${ }^{1}$ Hospital Regional Honorio Delgado
} 
influence of respiratory exercise in children with persistent asthma, obesity and overweight. Methods: A prospective and longitudinal case-control study was designed; It was carried out in the Pediatric Pneumology Outpatient Office of the Pediatrics Service of the Honorio Delgado Regional Hospital; from where 166 children from 6 to 11 years old were diagnosed with persistent bronchial asthma, which were divided equally into 2 groups; complying with the protocol, 25 children in the group that performed respiratory exercises and 52 children in the group that only received treatment with inhaled corticosteroids. Results: The male sex predominated in the participants, with an average age of 7.3 years, with a predominance of the moderate persistent asthma class and the majority with normal nutritional status; in the exercise-induced bronchoprovocation test, there was a similar improvement between the group that did a breathing exercise program and those that did not. Conclusion: Respi ratory exercise would improve lung function in overweight asthmatic children $(t=0.2835, p=0.777)$.

Keywords: overweight, obesity, bronchial asthma, children.

\section{INTRODUCCIÓN}

Siendo el Asma una de las enfermedades crónicas más frecuentes de la infancia, y a pesar de los muchos avances en el conocimiento de su fisiopatología y su tratamiento, aún hoy presenta una alta morbilidad y una mortalidad, a nivel mundial, con una prevale ncia entre el 8-10\% de (Blic, 2016) y una mortalidad, para el 2019, de 461000 muertes al año (GBD 2019 Diseases and Injuries Collaborators, 2020). Además, el asma puede ser clasificado de diferentes maneras, una es en base a la respuesta ante el ejercicio, la cual la divide en: a) la No inducida por el ejercicio físico y a) la Inducida por el ejercicio físico (AIE), ello debido a que el aire frío y seco que se inhala al realizar esfuerzos físicos hace que las vías respiratorias se estrechen, produciendo broncoconstriccción. Siendo, la práctica de actividad física uno de los estímulos provocadores de crisis en el individuo con Asma (Drobnic, 2001); además, la prevalencia del AIE es más frecuente en niños con asma (40-80\%) (Gerow, 2021). Es por ello que siendo el Asma bronquial un problema relevante en los niño, que en más del $80 \%$ es inducida por el ejercicio y, que el ejercicio adecuado no debe evitarse, sino por el contrario debe estimularse (AAP, 1989); es por ello que planteamos realizar el presente trabajo, y formulamos la siguiente pregunta: ¿Cuál es la influencia del efecto del ejercicio sobre la prueba de provocación bronquial basal en los niños de 6 a 11 años de edad con asma persistente en tratamiento ambulatorio de la Unidad de Neumología Pediátrica del Hospital Regional Honorio Delgado?; teniendo como objetivo: Determinar la influencia del ejercicio respiratorio en los niños con Asma Persistente con Obesidad y Sobrepeso.

\section{METODOLOGÍA}

El presente estudio se realizó en la Unidad de Neumología Pediátrica del Servicio de Pediatría del Hospital Regional Honorio Delgado, que se encuentra en la Ciudad de Arequipa. La información se recolectó entre el 1 de octubre del 2018 al 30 de setiembre del 2019. 
La población de estudio estuvo constituida por los niños de 6 a 11 años de edad que acudieron al Consultorio Externo de la Unidad de Neumología Pediátrica del Servicio de Pediatría del Hospital Regional Honorio Delgado con Asma Persistente, cuyos padres asintieron en participar y que cumplieron con los siguientes Criterios de inclusión: Antecedente de 3 episodios previos de sibilancias en los últimos 12 meses corroborados por un médico pediatra en Historia Clínica; tener prueba de provocación Bronquial Basal positiva, con caída del PEF mayor o igual al $11 \%$ del valor inicial (Lazo Velásquez, et al., 2005); niños que realizaron una Espirometría basal; ambos sexos; niños con asma persistente que usaron corticoides inhalados por lo menos 2 meses antes y corticoides sistémicos por lo menos 10 días antes de las pruebas; niños que tengan cualquier estado nutricional ; y se excluyó a los niños que no soplaron adecuadamente, cumplimiento del tratamiento antiinflamatorio al menos $80 \%$ del total de días., niños con patología respiratoria crónica, niños con tabaquismo pasivo, niños que vivan en una casa con riesgo de contaminación ambiental.

Fue una investigación, de casos y controles, prospectiva y longitudinal. Una vez solicitado el respectivo permiso a las autoridades del hospital, se recolectó la información a través de una Ficha de recolección de datos. Iniciaron el estudio166 niños, a un grupo de 88 niños que se les inició tratamiento con Corticoides inhalados de los cuales completaron 52 completaron el estudio y otro grupo de 88 niños a los que también se les inició tratamiento con Corticoides inhalados y adicionalmente se les agregó un programa de ejercicios físicos respiratorios basados en la Técnica Buteyko que consisten en realizar periodos de respiraciones lentas en las que se disminuye la frecuencia respiratoria combinado con periodos de apnea o mantenimiento de la respiración conocido como "control-pausa" (Cristancho, 2014), por lo menos 120 minutos a la semana, y por lo menos 2 veces a la semana, durante 12 semanas, este programa lo cumplieron 25 niños.

A todos los pacientes se les realizó una Espirometría Basal; y una Prueba de Broncoprovocación con el ejercicio basal; la cual se repitió luego de 12 semanas de tratamiento. La Espirometría basal, se realizó siguiendo una técnica estandarizada, y con equipo y persona bajo condiciones mínimas y con la técnica adecuada según la ATS (Agudelo, et al., 2016)).

La Prueba de provocación bronquial con ejercicio, se realizó con el fin de demostrar la existencia de hiperactividad bronquial al inicio, la cual fue cuantificada como positiva y negativa; bajo condiciones controladas en la Ciudad de Arequipa (Fadden, et al., 1979); como la intensidad del ejercicio afecta la respuesta, hay la necesidad de medir la intensidad del ejercicio, la frecuencia cardíaca fue mayor al $80 \%$ del máximo promedio para cada rango de edad. teniendo las siguientes condiciones previas: ausencia de síntomas de asma en el momento de inicio de la prueba, vestir ropa y calzado adecuados para el ejercicio. Se consideró como criterio de positividad un descenso del Flujo pico espiratorio (PEF) o Flujo espiratorio máximo (FEM) >= $11 \%$ del basal (Lazo-Velásquez et al., 2005) Los valores normales del PEF o FEM en Arequipa son (Loayza \& Recabarren, 2003):35 a) FEM teórico para varones normales= [Altura (cm) x5.56]-448; b) FEM teórico para mujeres= [Altura $(\mathrm{cm}) \times 4.63$ ]-338 La caída del FEM o PEF se calculó de la siguiente manera (Lazo-Velásquez et al., 2005): \% de Caída del FEM= ((FEM pre ejercicio)-menor FEM pos ejercicio))*100 FEM pre ejercicio. Luego de 12 semanas de tratamiento se repitió la prueba de provocación bronquial para poder comparar la variación de los resultados. 
Para la valoración del Estado nutricional, se pesó. y tallo al niño, y luego se procedió a calcular el Índice de masa corporal o IMC: Peso $(\mathrm{kg}) /$ talla $(\mathrm{m})$ 2, clasificando a los niños en base a las Tablas de OMS de IMC/ Edad, en sobrepeso ( $p 80$-p97), de la obesidad ( $p>97$ ), y con $p<10$ se consideró como de desnutrición (Segarra, et al., 2016).

Los pacientes asmáticos se clasificaron en base al número de exacerbaciones sufridas el último año se clasifica en intermitente o persistente y esta a su vez, en persistente leve, moderada o severa, de acuerdo a los criterios sugeridos por el Consenso Chileno (Calvo, 2010).

Una vez obtenidos los datos estos fueron procesados con estadística descriptiva, y para la comparación de los resultados dentro de cada grupo de estudio se usó la prueba de Wilcoxon y para la comparación de resultados entre los grupos se usó la prueba de la t.

\section{RESULTADOS}

Iniciaron el estudio dos grupos, cada grupo de 88 niños, a uno se les inició tratamiento con Corticoides inhalados $\mathrm{y}$ otro grupo de 88 niños tratamiento con Corticoides inhalados $\mathrm{y}$ adicionalmente un programa de ejercicios respiratorios basados en la Técnica de Buteyko, durante 12 semanas, de los 77 niños con asma ingresados al estudio la mayoría eran de sexo masculino (64.90\%), el mayor grupo estaba entre los 6 a 8 años (77.9\%), la mayoría tenía adecuado estado nutricional (39\%) pero era superado por el conjunto de niños con sobrepeso y obesidad (44/77), la mayoría no realizaron ejercicios respiratorios ( $N=52$ ) y predominaba el Asma bronquial persistente moderado (50.6\%), el asma en edad pediátrica es prevalente en varones, y como factor desencadenante cada vez más frecuente y que va de la mano con el incremento de niños con Sobrepeso/ Obesos.

Tabla 1

Características Clínicas de los pacientes CARACTERISTICAS

\begin{tabular}{llc} 
CARACTERISTICAS & $\mathrm{N}(\%)$ \\
\hline \multirow{3}{*}{ Sexo } & Masculino & $\mathbf{5 0}(\mathbf{6 4 . 9 0 )}$ \\
Estado Nutricional & Femenino & $27(35.10)$ \\
& Desnutricion & $3(3.90)$ \\
& Adecuado & $23(\mathbf{4 2 . 9 0 )}$ \\
& Sobrepeso & $21(27.90)$ \\
& Obesidad & $60(77.90)$ \\
\hline \multirow{2}{*}{ Edad } & 6 a 8 años & $17(22.10)$ \\
\hline \multirow{2}{*}{ Clase de Asma } & 9 a 11 años & $12(15.58)$ \\
& Leve & $39(50.65)$ \\
& Moderado & $26(33.77)$ \\
\hline & Severo & $25(32.50)$ \\
Ejercicios respiratorios & Si & $52(67.50)$ \\
\hline
\end{tabular}


Tabla 2

Estado Nutricional en los niños estudiados

\begin{tabular}{lcc}
\hline \multicolumn{1}{c}{ ESTADO } & SIN EJERCICIOS & CON EJERCICIOS \\
\multicolumn{1}{c}{ NUTRICIONAL } & $\mathbf{N} \%$ & $\mathbf{N} \%$ \\
\hline Obeso & $9(36)$ & $12(23)$ \\
Sobrepeso & $9(36)$ & $14(27)$ \\
Normal & $6(24)$ & $24(46)$ \\
Desnutrición & $1(4)$ & $2(4)$ \\
\hline Total & $25(100)$ & $52(100)$
\end{tabular}

\section{Gráfico 1}

Comparación de los valores de la Prueba de Provocación Bronquial con Ejercicio, al inicio y al Tercer mes del seguimiento

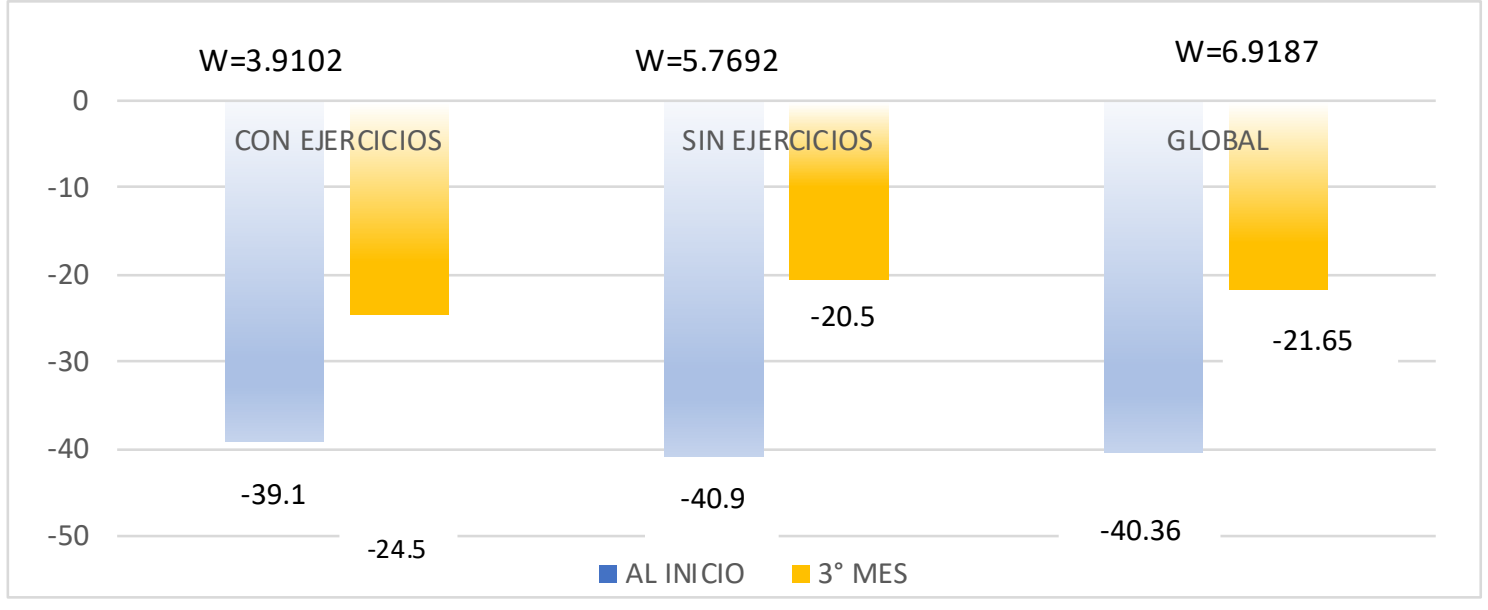

\section{Gráfico 2}

Comparación de la Variación de la Prueba de Broncoprovocación en relación a su Estado Nutricional, al Tercer mes del seguimiento

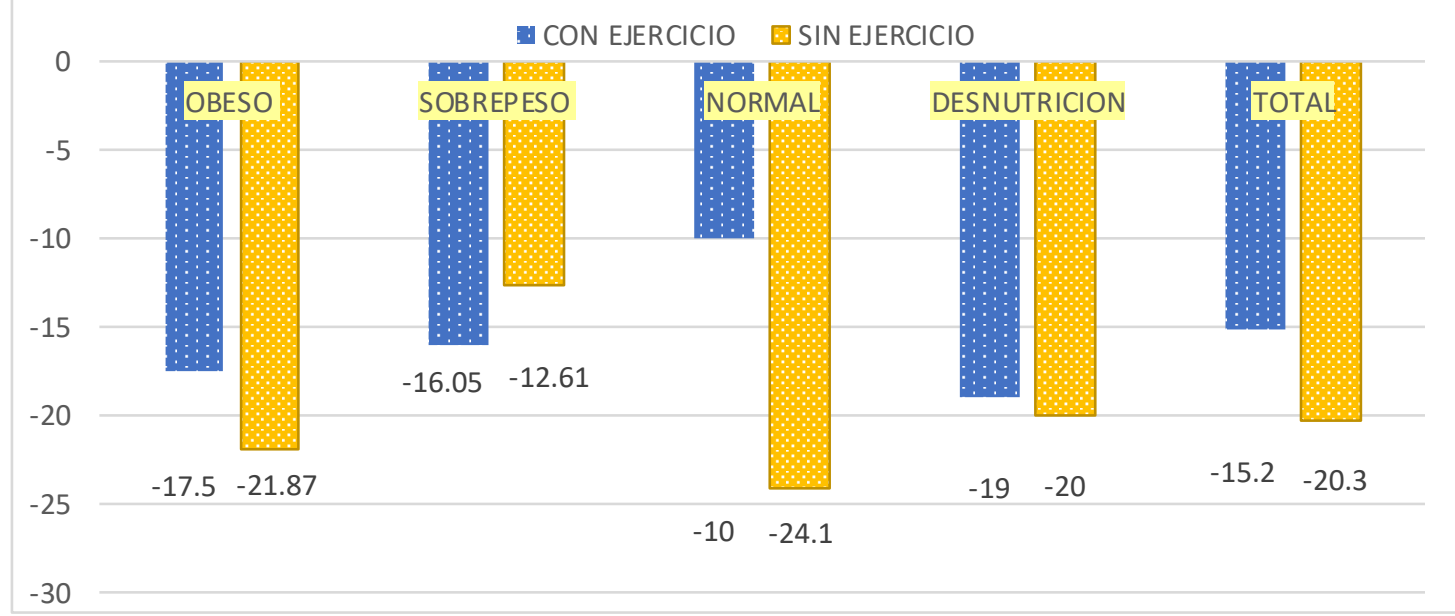




\section{DISCUSIÓN}

El menor diámetro de la vía aérea en los varones en edad pediátrica, similar al estudio de Chile donde el $60 \%$ eran de sexo masculino (Vicuña et al, 2007.), lo que lo hace un grupo de riesgo para presentar Asma y a menor edad (de 6 a 8 años); con una edad media de 7.3 años. El incremento del sedentarismo hace que la asociación Asma con Obesidad o Sobrepeso esté en aumento, lo cual es similar a otro estudio en Arequipa de Castro-Rodríguez et al. (2014), lo que incrementa la gravedad de su presentación sobre todo en los moderados o severos que también son predominantes en muestro estudio.

En lo que se refiere al Estado nutricional (Tabla 2), el mayor grupo era Adecuado en general y el grupo que realizo ejercicios similar a lo hallado por Castro-Rodríguez et al. (2014) en Arequipa donde los normales eran el mayor grupo; en el grupo de los que no realizaron ejercicios físicos el grupo de Obesos y con sobrepeso esto podría deberse al sedentarismo y al consumo de dietas altas en carbohidratos y lípidos por los niños lo que conlleva al aumento de peso y menor actividad física, que vale la pena separarlos como veremos posteriormente; el menor número de obesos y sobrepeso en el grupo de los que siguieron con el plan de ejercicios físicos se debería a que ellos tiene menor tolerancia al ejercicio y con ello la menor tolerancia a los ejercicios.

En el Grafico 1, Al evaluar la mejora en la prueba de bronco provocación con el ejercicio al tercer mes de tratamiento encontramos que la medición entre los portadores de Asma Bronquial pre tratamiento con corticoides inhalados eran menores que en el Post tratamiento, tanto en el Global, como en cada grupo, siendo esta variación estadísticamente significativa para los que realizaron ejercicios (Wilcoxon $=3.9102$ ), para los que no realizaron ejercicios $(p=0.000000$, Wilcoxon: 5.7692), como en el Global (Wilcoxon: 6.9187, $p=0.0000$ ). El tratamiento del Asma bronquial se basa en corticoides inhalados el cual mejora la función pulmonar de los niños; lo cual es similar a lo hallado en un estudio en Arequipa de Recabarren (2003) donde la variación en Global era de $-14.61 \%$ entre el pre y el post tratamiento.

En el grafico 2, al comparar la mejoría de la Prueba de Broncoprovocación con ejercicio según el Estado Nutricional, vemos que en los que realizaron ejercicios físico el grupo que tuvo menor mejoría fueron los que tenían un Adecuado estado nutricional, y en el global se ve mayor mejoría en los que no realizaron ejercicios físicos; similar a lo hallado por Coulson (2021) en un estudio en Arequipa (Molina, 2014) y los descrito por Cristancho (2014), y difiriendo con lo hallado por Jaakkola (2019); pero al analizar cada grupo hemos diferenciado el grupo de obesos y sobrepeso ya que difieren en resultados, contradiciendo lo dicho por Brüske, et al. (2014) que juntaba a los obesos y sobrepeso. En el grupo de Obesos, la mayor disminución se dio en los que no realizaron ejercicios $(t=1.1015, p=0,270)$ esto probablemente porque no solo el tratamiento con ejercicios serían suficientes, sino que adicionarles la baja de peso para tener una mejoría en la función pulmonar, como lo refiere Río Navarro, et al., (2011); pero en el grupo con Sobrepeso la mayor disminución se dio en los que realizaron ejercicios ( $t=0.2835, p=0.777)$, donde al parecer adicionar ejercicios físicos al tratamiento con estándar con Corticoides inhalados llevan a un mejor control de la enfermedad, contrario a lo descrito por Castro-Rodríguez, et al. (2001); en el grupo con estado nutricional adecuado también la mejoría fue mayor en los que no realizaron ejercicio $(t=2.2294, p=0.0257)$. 


\section{CONCLUSIÓN}

Los ejercicios respiratorios mejorarían la función pulmonar en los niños asmáticos con sobrepeso.

\section{REFERENCIAS}

de Blic, J. (2016). Asma infantil. EMC Pediatr. Sep;51(3):1-15. Spanish. doi: 10.1016/S12451789(16)77913-3. Epub 2016 Aug 23. PMID: 32288516; PMCID: PMC7147675. https://doi.org/10.1016/S1245-1789(16)77913-3

Global burden of 369 diseases and injuries in 204 countries and territories, 1990-2019: a systematic analysis for the Global Burden of Disease Study 2019 (2020). Lancet.; 396(10258):1204-22. https://www.thelancet.com/gbd/summaries

Drobnic, F. (2001). Asma inducida por esfuerzo. Madrid: Medical \& Marketing Communication.

Gerow, M., Bruner, J. (2021. Exercise Induced Asthma. In: Stat Pearls [Internet]. Treasure Island (FL): Stat Pearls Publishing; Jan. https://www.ncbi.nlm.nih.gov/books/NBK557554/

American Academy of Pediatrics (1989). Section on Allergy and Immunology and Section on Diseases of the Chest. Exercise and the asthmatic child. Pediatrics; 84: 392-393. https://pediatrics.aappublications.org/content/84/2/392.

Lazo, J., Recabarren, A., Montes, H. (2005). Evaluation of severity of bronchial asthma through and exercise bronchial challenge. Pediatr Pulmonol.; 40(5):457-62. https://www.ncbi.nlm.nih.gov/pubmed/16175592.

Cristancho, W. (2014). Fundamentos de fisioterapia respiratoria y ventilación mecánica. (3a ed.). Bogotá: Manual Moderno.

Posada S., Ricardo et al. (2016). Neumología Pediátrica. Bogotá: Distribuna.

McFadden, E. Jr., and Ingram, R. Jr. (1979). Exercise-induced asthma: observations on the initiating stimulus. The New England journal of Medicine; 301:763-760. https://www.ncbi.nlm.nih.gov/pmc/articles/PMC463084/.

Loayza, L., Recabarren, A. (2003). Curva normal de flujo espiratorio máximo en niños de Arequipa. Enfermedades de Tórax; 46: 40-43. http://sisbib.unmsm.edu.pe/BVRevistas /enfermedades_torax/v46_n1/curva.htm.

Clemente, S., Infante, D., Redecillas, S. (2016). Guía Nutrición Pediátrica Hospitalaria. Madrid Ergon.

Calvo, M. Clasificación del asma bronquial (2010). Neumología Pediátrica;1(2):66-68

Vicuña, P., Loza, C., Muñoz, P. \& Sanchez, I. (2007). Características clínicas de los niños asmáticos hospitalizados en un Servicio de Pediatría. Revista chilena de pediatría, 78(1), 29-34. https://dx.doi.org/10.4067/S0370-41062007000100004.

Castro-Rodríguez, J., Molina, R., Cáceres, M., Recabarren, A. (2014). Correlation between nitrites in induced sputum and asthma symptoms in asthmatic schoolchildren. Pediatr Pulmonol. Mar;49(3):214-20. doi: 10.1002/ppul.22843. Epub 2013 Jul 10. PMID: 23843321.

Recabarren, A., Parishuaña, E., Martínez, N. \& Esquía, G. (2017). Comparative assessment of bronchial asthma control level in patients aged 12 to 17 years old of EsSalud Arequipa. Horizonte Médico (Lima), 17(2), https://dx.doi.org/https://doi.org/10.24265/horizmed.2017.v17n2.03.

22-29. 
Recabarren, A., Portugal, K., Gutierrez, J. (2003). Comparación de las características clínicas del asma bronquial entre niños con sobrepeso/obesidad y niños eutróficos Inscritos en el Programa de Asma Bronquial del Hospital III Yanahuara EsSalud-Arequipa. Diagnóstico mayo-abril. Vol. №2:

17-22. http://www.fihu.org.pe/revista/numeros/2003/marabr03/17-22.html.

Molina, R. (2014). Efecto del sobrepeso y la obesidad sobre el tratamiento con Corticoides Inhalatorios en pacientes pediátricos con Asma bronquial persistente en el Hospital III Yanahuara ESSALUD. (Tesis Subespecialidad). Universidad Nacional de San Agustín, Arequipa.

Coulson, E., Carpenter, L. M., Georgia, T. E. \& Baptist, A. P. (2021). Breathing exercises in older adults with asthma: a blinded, randomized, placebo-controlled trial. The Journal of asthma: official journal of the Association for the Care of Asthma, 1-7. Advance online publication. https://doi.org/10.1080/02770903.2021.1936015

Jaakkola, J., Aalto, S., Hernberg, S., Kiihamäki, S. P., \& Jaakkola, M. S. (2019). Regular exercise improves asthma control in adults: A randomized controlled trial. Scientific reports, 9(1), 12088. https://doi.org/10.1038/s41598-019-48484-8

Brüske, I., Flexeder, C., Heinrich, J. (2014). Body mass index and the incidence of asthma in children. Current Opinion in Allergy and Clinical Immunology: April - Volume 14; Issue 2: 155-160. doi: 10.1097/ACI.0000000000000035.

Río Navarro, B., Berber, A. \& Sienra, J.J. (2011). Relación de la obesidad con el asma y la función pulmonar. Boletín médico del Hospital Infantil de México, 68(3), 171-183. http://www.scielo.org.mx/scielo.php?script=sci_arttext\&pid=S1665$11462011000300002 \&$ Ing=es\&tIng=es.

Castro-Rodríguez, J., Holberg, C., Morgan, W., et al. (2001). Increased incidence of asthma like symptoms in girls who become overweight or obese during the school years. Am J Respir Crit Care Med. May;163(6):1344-9. doi: 10.1164/ajrccm.163.6.2006140. PMID: 11371399. 